\title{
An Extensible Presentation Tool for Flexible Human-Information Interaction
}

\author{
Reinout Roels \\ Web \& Information Systems Engineering Lab \\ Vrije Universiteit Brussel \\ Pleinlaan 2, 1050 Brussels, Belgium \\ rroels@vub.ac.be
}

\author{
Beat Signer \\ Web \& Information Systems Engineering Lab \\ Vrije Universiteit Brussel \\ Pleinlaan 2, 1050 Brussels, Belgium \\ bsigner@vub.ac.be
}

\section{INTRODUCTION}

Nowadays, presentation tools such as PowerPoint and Keynote play an important role when transferring knowledge in educational or business settings. Nevertheless, a number of shortcomings of existing presentation tools in terms of the management, visualisation and navigation of content have been pointed out in literature [1]. Some of these limitations are based on the fact that slideware tools were originally used for the production of physical slides (e.g. transparencies) and existing solutions still simulate the affordances of physical slides. We investigate innovative forms to manage, visualise and navigate the content of a presentation, which, for example, includes the transclusion [2] or integration of content from other presentations and cross-media sources, as well as the non-linear navigation in a presentation. In order to explore and experiment with innovative human-information interaction techniques, we have developed the extensible MindXpres cross-media presentation platform. The modular architecture of MindXpres and its plug-in mechanism enable the reusability of content as well as the integration of new visualisation and interaction components which ultimately may improve the transfer of knowledge [3].

In this demo, we present the MindXpres crossmedia presentation tool shown in Figure 1 and highlight its innovative visualisation and navigation concepts. We further discuss the extensibility of MindXpres via its plug-in architecture and present and number of existing plug-ins. In order to highlight the extensibility and flexibility of our MindXpres presentation platform, we will also develop new plugins during the demo session based on requests from the audience.

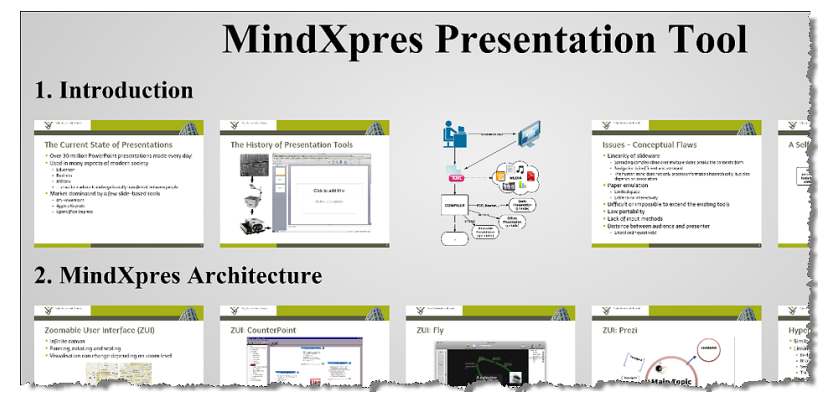

Figure 1: An HTML5-based zoomable MindXpres presentation

\section{MINDXPRES ARCHITECTURE}

An overview of the MindXpres architecture is given in Figure 2. Content is stored and structured in the MindXpres document format. A MindXpres document may contain various content elements as well as transclude external cross-media content. It can be created via a graphical authoring tool or be manually written in the Extensible Markup Language (XML). There is a clear separation between content and visualisation (e.g. font styles, colours or backgrounds), enabling the easy reuse of content or application of alternative themes.

The compiler transforms a MindXpres document into potentially different presentation formats. We can not only create dynamic interactive presentations but also static output formats (e.g. PDF for printing) can be supported. In addition, the compiler may resolve incompatibility issues and, for instance, automatically convert an unsupported video format. The result of the compilation step is a self-contained portable presentation bundle containing everything that is necessary for a presentation, in the form of a core presentation engine and the content to be presented. For dynamic presentations, the 


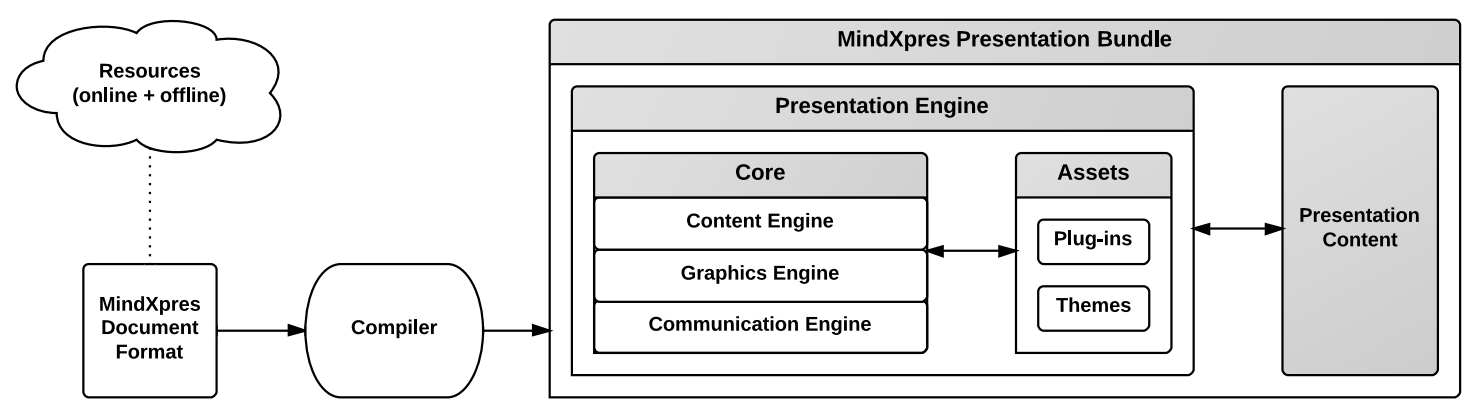

Figure 2: MindXpres architecture

presentation engine is based on HTML5, CSS3 and JavaScript. The core of the presentation engine consists of three modules. The content engine is responsible for processing the content and linking it to the corresponding visualisation plugins. The graphics engine abstracts all renderingrelated functionality and exposes this to the plug-ins, enabling a wide range of visualisations from classic slideshows to zooming user interfaces (ZUIs) as used by Prez 1 . The communication engine exposes a communication API that can be used by plugins (e.g. for the retrieval of information). In addition to the presentation content and core modules, the presentation bundle contains the set of plug-ins and themes that are referenced by the content.

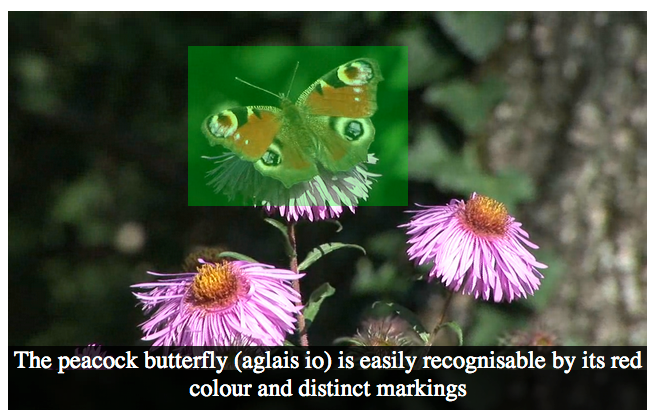

Figure 3: Video plug-in with highlighting and caption

When the content engine processes the content and encounters different content types, they are handed over to the corresponding plug-in which uses the graphics engine to visualise them. The themes contain aesthetic configurations either on a global or on a plug-in level. In order to provide a maximum of flexibility, all non-core MindXpres modules are implemented as plug-ins. We can distinguish three main categories of plug-ins:

Components form the basic building blocks of a presentation. These plug-ins handle the visualisation for specific content types such as text, images, bullet lists, graphs or videos. An example of an enhanced MindXpres video plug-in where specific parts of a video can be highlighted and time-synchronised text captions be added is shown in Figure 3

${ }_{1}^{1}$ http://prezi.com
Containers are responsible for grouping and organising components. An example of such a container is a slide. Each slide contains different content but there are also some reoccurring elements (e.g. the title or slide number) which can be abstracted in a higher level container and thereby taking some tedious work out of the user's hands. Another example is an image container visualising its content as a horizontally scrollable list of images. Note that we are not restricted to the slide format and content can be laid out in various other fashions.

Structures are high-level structures and layouts for components and containers. For instance, content can be scattered in a graph-like structure or it can be cleanly grouped in sections like a book as shown in Figure 1. These radically different ways of visualising and navigating content are abstracted by plug-ins and a user can easily switch between different presentation styles.

\section{CONCLUSION}

The presented extensible MindXpres presentation platform enables the rapid prototyping of novel information management, visualisation and navigation concepts. Our architectural choices and the plug-in based implementation of most functionality allow for innovation in human-information interaction, including the way presentations are created, the support of rich content types, innovative visualisations as well as new forms of non-linear navigation.

\section{REFERENCES}

[1] Edward R. Tufte. The Cognitive Style of PowerPoint: Pitching Out Corrupts Within. Graphics Press, July 2003.

[2] Theodor Holm Nelson. The Heart of Connection: Hypermedia Unified by Transclusion. Communications of the ACM, 38(8):31-33, August 1995.

[3] Andreas Holzinger, Michael D. Kickmeier-Rust, and Dietrich Albert. Dynamic Media in Computer Science Education; Content Complexity and Learning Performance: Is Less More? Educational Technology \& Society, 11(1):279-290, 2008. 